\title{
A Nonlinear Programming Approach to CDMA Multiuser Detection
}

\author{
Aylin Yener \\ WINLAB, Rutgers University \\ 73 Brett Road \\ Piscataway, NJ 08855-8060 \\ yener@winlab.rutgers.edu
}

\author{
Roy D. Yates \\ WINLAB, Rutgers University \\ 73 Brett Road \\ Piscataway, NJ 08855-8060 \\ ryates@winlab.rutgers.edu
}

\author{
Sennur Ulukus \\ AT\&T Labs-Research \\ Rm. 4-102, 100 Schulz Drive \\ Red Bank, NJ 07701-7033 \\ ulukus@research.att.com
}

\begin{abstract}
The optimum bit detector for multiuser CDMA systems has exponential complexity in the number of users. Many suboptimum receivers have been developed to achieve good performance with less complexity. In this work, we approximate the solution of the optimum multiuser detection problem using nonlinear programming relaxations. We observe that some popular suboptimum receivers correspond to relaxations of the optimal detection problem. In particular, one approximation method yields to iterative solutions which correspond to previously proposed heuristic nonlinear detectors. We identify the convergence properties of these iterative detectors. We also propose a relaxation that yields a receiver which we call the generalized MMSE detector. We give a simple iterative implementation of the detector. Its performance is evaluated and comparisons to other suboptimum detection schemes are given.
\end{abstract}

\section{Introduction}

It has been long known that the matched filter receiver designed for a single user Gaussian channel is not optimum for the multiple-user CDMA channel [13]. Further, optimum detection of multiple users' bits has been shown to be an NP hard problem [12]. This observation resulted in the development of many suboptimum receivers that have reasonable complexity with near-optimum performance $[2,5,6,10]$. These suboptimum receivers have been motivated by several criteria. Among the most popular linear detectors are the decorrelator [5] and the MMSE receiver [6]. The decorrelator suppresses the multiple access interference totally while enhancing the Gaussian noise and is the optimum detector if the received powers of the users are unknown at the receiver. The MMSE receiver [6] gives the minimum mean squared error between the filter output and the transmitted bit, and also maximizes the output signal to interference ratio. Both detectors are optimum when no noise is present.

Our aim in this study is to approach the optimum multiuser detection problem from a nonlinear programming point of view. The original optimum multiuser detection problem (OMUD) is a $0-1$ quadratic program for which there exists no efficient algorithm. The general approach in the presence of such hardship is to approximate the solution by working on an easier problem that can be solved efficiently. The easier problem to be solved is a relaxation of the original problem. The relaxed solution is then mapped to the solution set of the original problem, ideally arriving at a near optimum solution.

Using nonlinear programming approach, we see that some popular suboptimum detectors are relaxed solutions to the optimum detection problem. This approach helps us understand the previously unidentified convergence properties of some known iterative nonlinear detectors. Furthermore, a new relaxation method is proposed that results in a simple iterative detector whose performance is then evaluated.

\section{OMUD and its Relaxations}

We consider a synchronous CDMA system employing BPSK modulation. The received signal is given by

$$
r(t)=\sum_{i=1}^{N} \sqrt{q_{i}} a_{i} s_{i}(t)+n(t)
$$

where $N$ is the number of users, $q_{i}$ and $a_{i}$ are received power and the transmitted bit ( \pm 1 equiprobably) of the $i$ th user and $n(t)$ is the additive white Gaussian noise (AWGN) process with power spectral density $\sigma^{2}$. The received signal vector at the output of the matched filters is a sufficient statistic for the multiuser detection problem and is given by

$$
y=\Gamma \Lambda a+n
$$

In (2), $\boldsymbol{\Gamma}$ is the nonnegative definite cross correlation matrix with $\Gamma_{i j}=\int_{0}^{T} s_{i}(t) s_{j}(t) d t, \boldsymbol{\Lambda}$ is a diagonal matrix containing the users' received amplitudes $\Lambda_{i i}=\sqrt{q_{i}}, \boldsymbol{a}$ is the vector containing the information bits of the users and $\boldsymbol{n}$ is a zero mean Gaussian random vector with auto covariance matrix $E\left[\boldsymbol{n} \boldsymbol{n}^{\top}\right]=\sigma^{2} \boldsymbol{\Gamma}$. 
The aim of multiuser detection is to recover the information bits, $\boldsymbol{a}$. The solution of the optimum multiuser detection problem (OMUD) [11] employs the maximum likelihood estimate $\boldsymbol{a}$ given $\boldsymbol{y}$. Specifically,

$$
\boldsymbol{a}^{*}=\arg \min _{\boldsymbol{a} \in\{-1,1\}^{N}} \boldsymbol{a}^{\top} \boldsymbol{R} \boldsymbol{a}-2 \boldsymbol{a}^{\top} \boldsymbol{\Lambda} \boldsymbol{y}
$$

where $\boldsymbol{R}=\boldsymbol{\Lambda} \boldsymbol{\Gamma} \boldsymbol{\Lambda}$ with $R_{i j}=\sqrt{q_{i}} \sqrt{q_{j}} \Gamma_{i j}$.

Although it has been shown recently that certain special $\boldsymbol{R}$ structures allow construction of polynomial time algorithms to find the optimum solution [9], the problem for general correlation matrices remains NP hard and one can find the optimum $\boldsymbol{a}$ only by exhaustive search of $2^{N}$ candidate vectors.

In this work, we will concentrate on cases where the signatures of the users are independent and $\boldsymbol{\Gamma}$ and hence $\boldsymbol{R}$ are positive definite. In this case, the objective (3) is strictly convex in $\boldsymbol{a}$ and has a well defined unique minimizer over a convex set. Thus, we can find solutions by relaxing the constraint set -which in the original problem contains only the corners of the unit hypercube- such that the resulting "relaxed" constraint set is convex. Figure 1 shows the different relaxed constraint sets for the two-user case. Note that the requirement is that for each relaxation the relaxed constraint set contains the feasible set of the original problem. The solution can then be mapped to the feasible set of the original problem by taking the sign of each component of the relaxed solution vector (bits are equiprobably \pm 1 ).

\section{Decorrelator}

We first consider the simplest relaxation, where the feasible set is relaxed to contain the $N$ dimensional space $\mathcal{R}^{N}$.

$$
\min _{\boldsymbol{a} \in \mathcal{R}^{N}} \boldsymbol{a}^{\top} \boldsymbol{R} \boldsymbol{a}-2 \boldsymbol{a}^{\top} \boldsymbol{\Lambda} \boldsymbol{y}
$$

This problem has a unique minimum at

$$
\hat{\boldsymbol{a}}=\boldsymbol{R}^{-1} \boldsymbol{\Lambda} \boldsymbol{y}=\boldsymbol{a}+\boldsymbol{\Lambda}^{-1} \boldsymbol{\Gamma}^{-1} \boldsymbol{n}
$$

Taking the sign of the solution vector yields the well known decorrelating detector [5].

\section{Soft Interference Cancellation}

The constraint set of the optimum multiuser detection problem (3) consists of the corner points of the unit hypercube. An effective approximation method is to relax the constraint set to cover to whole hypercube and use nonlinear programming algorithms to find the solution of the new convex programming problem [3]. The relaxed problem is:

$$
\boldsymbol{a}^{*}=\arg \min _{\boldsymbol{a} \in[-1,1]^{N}} \boldsymbol{a}^{\top} \boldsymbol{R} \boldsymbol{a}-2 \boldsymbol{a}^{\top} \boldsymbol{\Lambda} \boldsymbol{y}
$$

The above optimization yields the optimum detector under certain conditions. Consider the case where the transmit

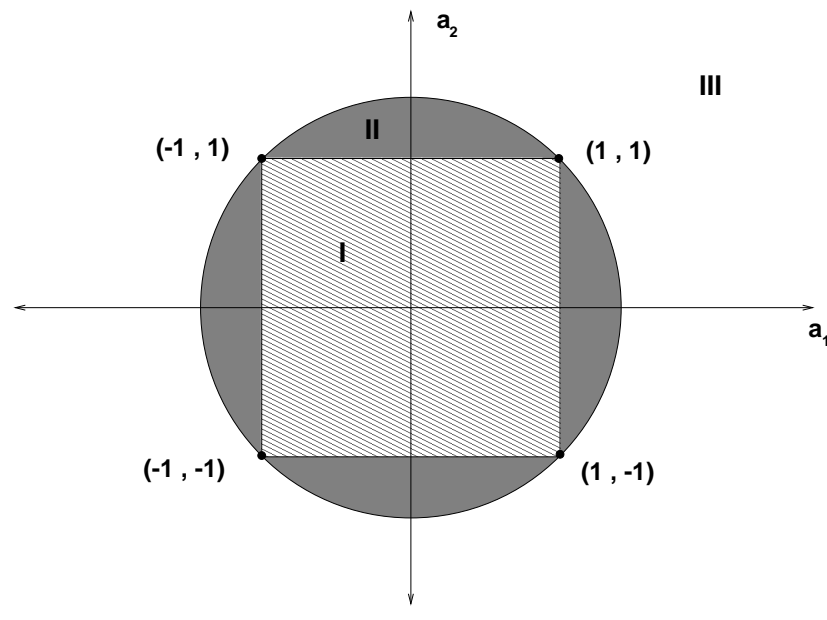

Figure 1. Relaxed constraint sets for the two user system which yield the following detectors: I - Soft Interference Canceller (Section 4), I+II - Generalized MMSE (Section 5), I+II+III - Decorrelator (Section3)

powers $\left\{p_{i}\right\}$ of the users are known but the uplink gains, $h_{i} \in[0,1]^{N}$, are random, i.e. the receiver only knows that the received power of user $i$ is in $\left[0, p_{i}\right]$. Defining $\boldsymbol{\Upsilon}$ and $\boldsymbol{H}$ as the diagonal matrices with $\Upsilon_{i i}=\sqrt{p_{i}}$ and $H_{i i}=\sqrt{h_{i}}$ $\left(\sqrt{q_{i}}=\sqrt{p_{i}} \sqrt{h_{i}}\right)$, the joint maximum likelihood estimation problem for the uplink gains and the bits becomes

$$
\min _{a_{i} \in\{-1,1\}, h_{i} \in[0,1] \forall i} \boldsymbol{a}^{\top} \boldsymbol{H} \Upsilon \boldsymbol{\Gamma} \Upsilon \boldsymbol{H} \boldsymbol{a}-2 \boldsymbol{a}^{\top} \boldsymbol{H} \Upsilon \boldsymbol{y}
$$

Defining $\tilde{a}_{i}=a_{i} \sqrt{h_{i}}$ and $\tilde{\boldsymbol{R}}=\boldsymbol{\Upsilon} \boldsymbol{\Gamma} \Upsilon$ yields the optimization problem

$$
\tilde{\boldsymbol{a}}^{*}=\arg \min _{\tilde{\boldsymbol{a}} \in[-1,1]^{N}} \tilde{\boldsymbol{a}}^{\top} \tilde{\boldsymbol{R}} \tilde{\boldsymbol{a}}-2 \tilde{\boldsymbol{a}}^{\top} \mathbf{\Upsilon} \boldsymbol{y}
$$

which is identical to (6). The joint maximum likelihood estimates of the uplink gains and the information bits are:

$$
\sqrt{h_{i}^{*}}=\left|\tilde{a}_{i}^{*}\right| \quad a_{i}^{*}=\operatorname{sgn}\left(\tilde{a}_{i}^{*}\right) \quad i=1, \ldots, N
$$

Now we consider the implementation of the receiver given by (6). Since the optimization is a convex minimization over a convex set, the unique fixed point is the minimum. However, the optimum point does not have a closed form and one should use iterative methods to get to the solution. One class of iterative methods that can be used are the constrained gradient methods. Further, the simplicity of the constraint set, i.e. the fact that it has a cartesian product form, enables us to define special iterative projection algorithms [1]. In particular, the following two algorithms, the nonlinear Gauss-Seidel and the nonlinear Jacobi algorithms respectively, converge to the minimum of (6) under certain 
conditions. Let $g\left(a_{1}, \ldots, a_{N}\right)=\boldsymbol{a}^{\top} \boldsymbol{R} \boldsymbol{a}-2 \boldsymbol{a}^{\top} \boldsymbol{\Lambda} \boldsymbol{y}$ denote the function to be minimized. In the Gauss-Seidel iteration, $a_{i}(t+1)$ is found by

$\arg \min _{x \in[-1,1]} g\left(a_{1}(t+1), \cdots, x, a_{i+1}(t), \cdots, a_{N}(t)\right)$

while in the Jacobi iterations, $a_{i}(t+1)$ is

$$
\arg \min _{x \in[-1,1]} g\left(a_{1}(t), \cdots, x, a_{i+1}(t), \cdots, a_{N}(t)\right)
$$

respectively, where $t$ is the stage (iteration) index. Both algorithms optimize one variable at a time to get to the optimum point of (6); however (9) uses the current stage estimates of some of the users while (10) allows a parallel implementation. Through a straightforward derivation, it can be shown that the above iterations yield the following twostep algorithms. For each user $i$, the first step for the GaussSeidel iteration is,

$\hat{x}(t+1)=$

$\frac{1}{\sqrt{q_{i}}}\left(y_{i}-\sum_{j=1}^{i-1} \sqrt{q_{j}} \Gamma_{j i} a_{j}(t+1)-\sum_{j=i+1}^{N} \sqrt{q_{j}} \Gamma_{j i} a_{j}(t)\right)$

and the first step for the Jacobi iteration is,

$$
\hat{x}(t+1)=\frac{1}{\sqrt{q_{i}}}\left(y_{i}-\sum_{j=1, j \neq i}^{N} \sqrt{q_{j}} \Gamma_{j i} a_{j}(t)\right)
$$

The second step for both algorithms is

$$
a_{i}(t+1)= \begin{cases}-1, & \hat{x}(t+1)<-1 \\ \hat{x}(t+1), & -1 \leq \hat{x}(t+1) \leq 1 \\ 1, & \hat{x}(t+1)>1\end{cases}
$$

At each stage, to get the estimate of each user's bit, both receivers use soft estimates of the bits to reconstruct the interference and subtract this estimate from the user's matched filter output, scale the result by the amplitude of the user and project onto $[-1,1]$. The difference between the two is that while the Gauss-Seidel algorithm uses the available current stage estimates of the users, i.e. feedback from a group of users whose bit estimates are already computed, the Jacobi algorithm uses only bit estimates from the previous stage.

Convergence of the Gauss-Seidel algorithm is easily established using Proposition 3.9 of Section 3.3 of [1] which says that if the convex function to be optimized is strictly convex in each of its variables and the constraint set has a Cartesian product form, the algorithm will converge to the unique minimum. Since we have a positive definite $\boldsymbol{\Gamma}$, the function $g(\boldsymbol{a})$ is convex in $\boldsymbol{a}$ and is strictly convex in each variable when the values of the other components of $\boldsymbol{a}$ are held constant. The convex set $X=[-1,1]^{N}$ is in Cartesian product form. The convexity of $g(\boldsymbol{a})$ ensures the uniqueness of the convergence point which is the global minimum.
Corollary 1 For a CDMA system with linearly independent signature sequences, the Gauss-Seidel algorithm (the successive soft interference canceller) always converges to the minimizer of (6).

Establishing the convergence for the Jacobi algorithm requires a little more effort. Convergence can be guaranteed under certain contraction assumptions as indicated by Proposition 3.10 of Section 3.3 of [1] which is given below.

Theorem 1 Let $g: \mathcal{R}^{N} \rightarrow \mathcal{R}$ be continuously differentiable, let $\gamma$ be a positive scalar, and suppose that the mapping $T: X \rightarrow \mathcal{R}^{N}$, defined by $T(\boldsymbol{x})=\boldsymbol{x}-\gamma \nabla g(\boldsymbol{x})$, is a contraction with respect to the block maximum norm $\|\boldsymbol{x}\|=$ $\max _{i}\left\|x_{i}\right\|_{i} / w_{i}$, where each $\|\cdot\|_{i}$ is the Euclidean norm on $\mathcal{R}^{n_{i}}$ and each $w_{i}$ is a positive scalar. Then, there exists a unique vector $\boldsymbol{x}^{*}$ which minimizes $g$ over $X$. Furthermore, the sequence $\boldsymbol{x}(t)$ generated by either of the Gauss-Seidel and the Jacobi algorithms converges to $\boldsymbol{x}^{*}$ geometrically.

The necessary and sufficient condition for $T(\boldsymbol{x})$ to be a contraction mapping for our problem such that Theorem 1 is valid can be shown to be

$$
\|\boldsymbol{I}-\gamma \boldsymbol{R}\|_{\infty}^{w}<1
$$

where, for any matrix $\mathbf{A},\|\mathbf{A}\|_{\infty}^{w}=\max _{i} \frac{1}{w_{i}} \sum_{j}\left|A_{i j}\right| w_{j}$. For a small enough $\gamma,(14)$ is equivalent to

$$
\max _{i}\left(R_{i i}-\sum_{j \neq i}\left|R_{i j}\right| \frac{w_{j}}{w_{i}}\right)>0
$$

Define the matrix $\overline{\boldsymbol{\Gamma}}$ with $\bar{\Gamma}_{i j}=\left|\Gamma_{i j}\right|$, if $i \neq j$, and $\bar{\Gamma}_{i i}=0$. Then, it can be shown that for some $\bar{w},(15)$ is equivalent to

$$
\|\overline{\boldsymbol{\Gamma}}\|_{\infty}^{\bar{w}}<1
$$

For a given $\Gamma$, it may be difficult to check this condition for all possible $\bar{w}$ values. The following equivalent condition that is independent of the particular norm can be found by using Corollary 6.2 in Section 2.6 of [1].

$$
\rho(\overline{\boldsymbol{\Gamma}})<1
$$

where $\rho(\boldsymbol{A})$ denotes the maximum eigenvalue of $\boldsymbol{A}$.

It is interesting to note that conditions (16) and (17) are satisfied for a system where users' signatures are shifted versions of a basic $m$-sequence. In this case, $\Gamma_{i j}=-1 / G$, $i \neq j$, and $\rho(\overline{\boldsymbol{\Gamma}})=(N-1) / G . \rho(\overline{\boldsymbol{\Gamma}})<1$ as long as $N \leq G$ which by definition is the case. Note that choosing $w_{i}=1$ for all $i,(16)$ reduces to a diagonal dominance condition which is a sufficient condition for convergence and is also equivalent to $(N-1) / G<1$ for $m$-sequences. Thus, if $m$-sequences are used, both Jacobi and Gauss-Seidel algorithms, i.e., parallel and successive interference cancellers, converge to the minimizer of (6). 
In general, it takes more than one iteration for either algorithm to converge and thus the resulting receivers are multi-stage receivers. Multi-stage receivers are familiar in multiuser detection. [10] proposes using hard decision bit estimates to reconstruct and subtract the interference for each user. The receiver is implemented in a parallel fashion as in (12) and is not convergent. [8] proposes a class of receivers based on the SAGE algorithm, one of which is the successive multistage receiver (11) and argues that the SAGE based hard decision multistage receiver is convergent even when its parallel counterpart is not. The soft decision versions of these multistage receivers, i.e. (11) and (12), are proposed in [8] and [14]. They are termed as receivers with linear clippers. By representing these receivers in the form of iterative nonlinear programming algorithms, we have shown that both these soft decision receivers, i.e. the parallel and the successive soft multi-stage interference cancellers, if they converge, converge to the same point which is the minimizer of (6). Typically, Gauss-Seidel type iterations have faster convergence since they use the newest estimates. On the other hand, Jacobi type iterations can be executed in a completely parallel fashion since they do not require feedback from the current stage estimate of any user. Note that, if Theorem 1 is valid, any combination of the two algorithms also converges to the minimum of (6), i.e. some users can use the successive soft multi-stage receivers and others can use the parallel soft multi-stage receivers.

It is worthwhile to note that, one can implement the decorrelator given by (5) iteratively. Gauss-Seidel and Jacobi algorithms that converge to (5) can be found to be the algorithms derived in this section without the second stage $[-1,1]$ clippers. The convergence conditions are identical to those discussed in this section. It is also possible to derive Gauss-Seidel and Jacobi iterations that converge to the MMSE detector [6] which estimates the bits by taking the sign of $\overline{\boldsymbol{a}}=\left(\boldsymbol{\Gamma}+\sigma^{2} \boldsymbol{\Lambda}^{-2}\right)^{-1} \boldsymbol{y}$. It can be shown that the resulting algorithms differ from (11) and (12) only in the scaling factor. Specifically, one has to replace $1 / \sqrt{q_{i}}$ with $\sqrt{q_{i}} /\left(q_{i}+\sigma^{2}\right)$.

Finally, we should emphasize that the implementations discussed here are not the unique way of solving for the minimizer of (6). There are other nonlinear programming methods that yield iterative algorithms whose bit error rate performance matches that of the soft interference cancellers.

\section{Generalized MMSE Detector}

The constraint on each $a_{i} \in\{-1,1\}$ is equivalent to $a_{i}^{2}=1$ which implies $\boldsymbol{a}^{T} \boldsymbol{a}=N$ at any feasible point for OMUD. Relaxing this set to $\boldsymbol{a}^{T} \boldsymbol{a} \leq N$ results in:

$$
\min _{\boldsymbol{a}^{\top} \boldsymbol{a} \leq N} \boldsymbol{a}^{\top} \boldsymbol{R} \boldsymbol{a}-2 \boldsymbol{a}^{\top} \boldsymbol{\Lambda} \boldsymbol{y}
$$

Since (18) minimizes of a convex function over a convex set, it has a unique minimum and iterative algorithms such as gradient descent can be employed to find this minimum [7]. Further, the convex duality theorem [7, Theorem 14.6] ensures that no duality gap exists and one can solve for the dual problem instead. Since (18) has a single constraint, there is only one dual variable. Thus, a simpler iterative algorithm can be found by solving the dual problem as outlined below.

The Lagrangian dual function can be expressed as

$$
\mathcal{L}(\boldsymbol{a}, \lambda)=\boldsymbol{a}^{\top} \boldsymbol{R} \boldsymbol{a}-2 \boldsymbol{a}^{\top} \boldsymbol{\Lambda} \boldsymbol{y}+\lambda\left(\boldsymbol{a}^{\top} \boldsymbol{a}-N\right)
$$

which is to be maximized over $\boldsymbol{a}$ and $\lambda \geq 0$. Solving for $\boldsymbol{a}$ in terms of $\lambda$ and substituting back we arrive at:

$$
\max _{\lambda \geq 0}-\boldsymbol{y}^{\top} \boldsymbol{\Lambda}(\boldsymbol{R}+\lambda \boldsymbol{I})^{-1} \boldsymbol{\Lambda} \boldsymbol{y}-\lambda N
$$

which is a one-dimensional optimization problem and can be solved with a variety of iterative algorithms [7]. A simple unconstrained gradient descent algorithm is guaranteed to converge for a small enough step size $\mu$ which can then be projected onto the positive axis. The algorithm is

$$
\bar{\lambda}(t+1)=\bar{\lambda}(t)+\mu\left(\boldsymbol{y}^{\top} \boldsymbol{\Lambda}(\boldsymbol{R}+\bar{\lambda}(t) \boldsymbol{I})^{-2} \boldsymbol{\Lambda} \boldsymbol{y}-N\right)
$$

which converges to $\bar{\lambda}$. The maximizer of (20) is given by

$$
\lambda^{*}=\max (0, \bar{\lambda})
$$

Then, the unique minimizer of (18) can be found to be

$$
\boldsymbol{a}^{*}=\left(\boldsymbol{R}+\lambda^{*} \boldsymbol{I}\right)^{-1} \boldsymbol{\Lambda} \boldsymbol{y}=\boldsymbol{\Lambda}^{-1}\left(\boldsymbol{\Gamma}+\lambda^{*} \boldsymbol{\Lambda}^{-2}\right)^{-1} \boldsymbol{y}
$$

The form of this solution whose sign is the estimate of the bit vector is also familiar because of its similarity to the MMSE detector [6]. We term the relaxation (18) the generalized MMSE (GMMSE) solution. When $\lambda^{*}=\sigma^{2}$, (23) reduces to the MMSE detector. Note that finding the GMMSE solution results in a nonlinear multiuser detector in contrast to the MMSE detector. On the other hand, the knowledge of the noise power value $\left(\sigma^{2}\right)$ is not necessary for the GMMSE detector whereas the MMSE detector requires this knowledge if training or blind adaptation is not desired $[4,6]$.

The GMMSE detector is also an iterative detector since $\lambda^{*}$ has to be found iteratively. However, since the iterations are in one dimension, they are expected to converge quickly compared to multidimensional algorithms.

\section{Results and Discussion}

Since the probability of bit error expressions are not analytically tractable for arbitrary number of users and iterations, we have simulated the bit error performance of the detectors investigated in this work. The first system simulated is an $N=7$ user system with processing gain $G=7$ that uses $m$-sequences. Figure 2 shows the probability of bit error for one user when that user has $0 \mathrm{~dB}$ SNR and all the interferers have a common SNR that is varied. All iterative 


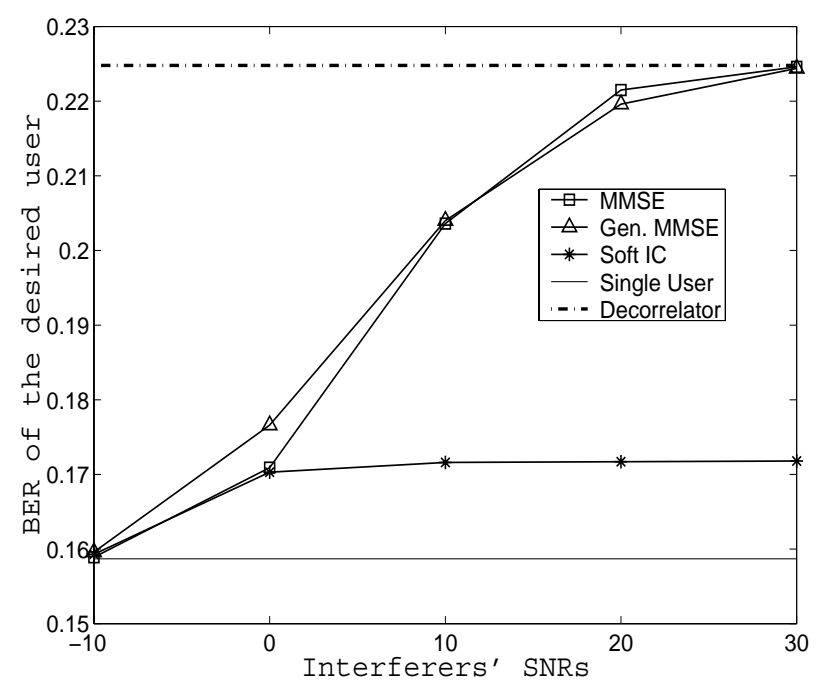

Figure 2. Comparison of error probabilities of nearoptimum multiuser detectors: Near-far scenario (Desired user at $0 \mathrm{~dB} \mathrm{SNR}) . G=7, N=7$, M-sequences.

detectors (multistage soft cancellers and the GMMSE) are evaluated at their convergence points. The soft interference cancellers $((11),(12),(13))$ have almost invariable performance versus interference strength. We note that the performance of the GMMSE detector is similar to that of the linear MMSE detector. In particular, we observe that the GMMSE detector has the same trend of approaching the decorrelator performance as the MMSE detector as the interference dominates the noise. We have also simulated an $N=4$ user system with processing gain $G=7$ that uses Gold sequences and observed similar results (Figure 3).

In this paper, we have shown that many popular suboptimum detectors are devices that attempt to approximate the solution of the joint minimum bit error rate detector (OMUD). Although it is analytically hard to characterize exactly how closely they approximate the OMUD cost function, we have observed that they achieve near-optimum cost values. Consequently, the near-optimum bit error rate performances of these detectors are not surprising. We have identified the convergence conditions of multistage soft interference cancellers. We have also proposed and devised a simple iterative nonlinear detector with similar performance to the MMSE detector. It can be used in scenarios where adaptive or blind adaptive detection is not suitable -say due to delay constraints- and the ambient noise power is unknown.

\section{References}

[1] D. Bertsekas and J. Tsitsiklis. Parallel and Distributed Computation. Prentice-Hall, 1989.

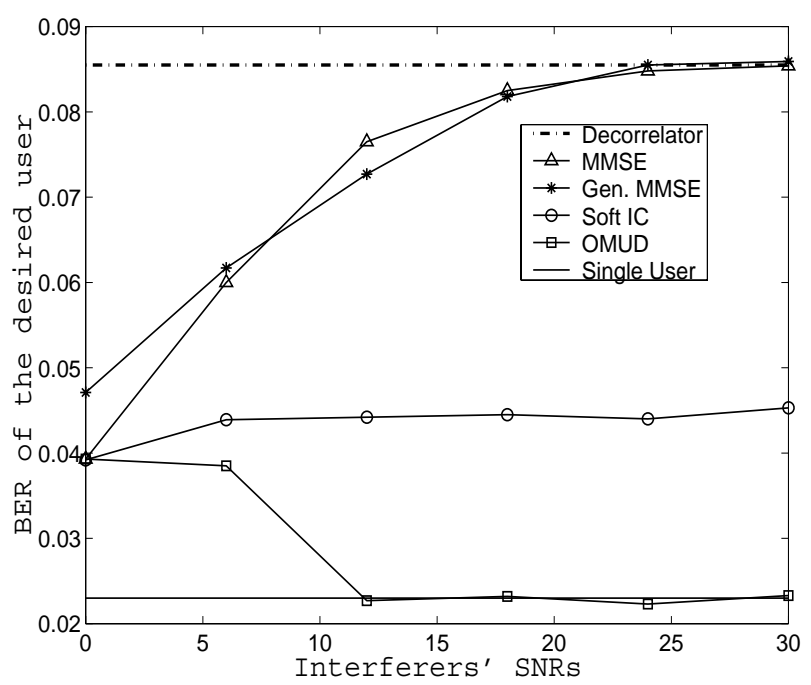

Figure 3. Comparison of error probabilities of nearoptimum multiuser detectors: Near-far scenario (Desired user at $6 \mathrm{~dB} \mathrm{SNR}) . G=7, N=4$, Gold sequences.

[2] A. Duel-Hallen. Decorrelating decision-feedback multiuser detector for synchronous code-division multiple-access channels. IEEE Trans. Comm., 41(2):285-290, 1993.

[3] P. Hansen. Methods of nonlinear 0-1 programming. Annals of Discrete Mathematics 5: Discrete Optimization II, 1979. Noth Holland.

[4] M. Honig, U. Madhow, and S. Verdú. Blind adaptive multiuser detection. IEEE Trans. Info.Th., 41(4):944-960, 1995.

[5] R. Lupas and S. Verdú. Linear multiuser detectors for synchronous code-division multiple-access channels. IEEE Trans. Info.Th., 35(1):123-136, 1989.

[6] U. Madhow and M. L. Honig. MMSE interference suppression for direct-sequence spread-spectrum CDMA. IEEE Trans. Comm., 42(12):3178-3188, 1994.

[7] S. G. Nash and A. Sofer. Linear and Nonlinear Programming. Preprint, 1995.

[8] L. B. Nelson and H. V. Poor. Iterative multiuser receivers for CDMA channels: An EM-based approach. IEEE Trans. Comm., 44(12):1700-1710, 1996.

[9] S. Ulukus and R. D. Yates. Optimum multiuser detection is tractable for synchronous CDMA systems using msequences. IEEE Comm. Letters, 2(4):89-91, 1998.

[10] M. K. Varanasi and B. Aazhang. Multistage detection in asynchronous code-division multiple-access communications. IEEE Trans. Comm., 38(4):509-519, 1990.

[11] S. Verdú. Minimum probability of error for asynchronous gaussian multiple-access channels. IEEE Trans. Info. Th., 32:85-96, 1986.

[12] S. Verdú. Computational complexity of multiuser detection. Algorithmica, 4(4):303-312, 1989.

[13] S. Verdú. Multiuser Detection. Cambridge U. Press, 1998.

[14] X. Zhang and D. Brady. Asymptotic multiuser efficiencies for decision-directed multiuser detectors. IEEE Trans. Info. Th., 44(2):502-515, 1998. 